\title{
High-Selectivity Single-Ended and Balanced Bandpass Filters Using Ring Resonators and Coupled Lines Loaded with Multiple Stubs
}

\author{
Kai Da Xu, Zekai Luo, Yanhui Liu, and Qing Huo Liu
}

\begin{abstract}
High-selectivity single-ended and balanced bandpass filters (BPFs) using dual-mode ring resonators and coupled lines loaded with multiple stubs are proposed in this paper. With the help of the loaded short-circuited and open-circuited stubs, six deep transmission zeros (TZs) from 0 to $2 f_{0}\left(f_{0}\right.$ : center frequency of the passband) can be realized in both of single-ended and balanced BPFs to improve the stopband suppressions. The functions of the loaded short/open stubs and calculated analysis of TZs' positions have been presented. For further demonstration, two examples of single-ended BPF and balanced BPF with high common-mode suppression are designed and fabricated, whose center frequencies are both at 2.1 GHz. Their measured 3-dB fractional bandwidths are $23.7 \%$ and $24.7 \%$ (differential-mode), respectively. The simulated results and measurements of these two filters are in good agreement.
\end{abstract}

Keywords-Balanced bandpass filters, high-selectivity filters, ring resonators, parallel-coupled lines, transmission poles, transmission zeros.

\section{INTRODUCTION}

High-performance components and circuits are extensively desirable in the modern radio-frequency and microwave wireless communication systems. These components mainly include active circuits such as power amplifiers [1] and passive circuits such as power dividers [2] and bandpass filters (BPFs) [3]-[5]. The design of the BPFs is indispensable to meet the specific demands of consumer electronics and industrial applications. Recently, many planar BPFs with low insertion losses in the passbands and high out-of-band rejections have been reported using signal-interference techniques [6]-[7], stub loaded techniques [8]-[9], substrate integrated waveguide [10] and so on.

Additionally, balanced BPFs have more advantages of noise immunity and low electromagnetic interference in wireless communication systems compared with their single-ended counterparts [11]. Numerous planar balanced BPFs with differential-mode (DM) filtering characteristic and common-mode (CM) suppression have been proposed in

This work was supported in part by the National Natural Science Foundation of China under Grant 61601390 and in part by the Shenzhen Science and Technology Innovation Project under Grant JCYJ20170306141249935.

K. D. Xu, Z. Luo, and Y. Liu are with the Department of Electronic Science, Xiamen University, Xiamen 361005, and also with the Shenzhen Research Institute of Xiamen University, Shenzhen 518057, China.

Q. H. Liu is with the Department of Electrical and Computer Engineering, Duke University, Durham, NC 27708, USA.
[12]-[21]. In [12], a fourth-order differential BPF using four folded stepped-impedance resonators is presented with extended upper stopband. Moreover, balanced filters with different configurations are introduced by using multilayer structure [13], ring resonators [14], coupled line scheme [15], stub loaded structures [16][17], and combined structures of coupled lines and stubs [18]-[20].

In this paper, a new high-selectivity single-ended BPF using dual-mode ring resonator and coupled lines loaded with open and short stubs is proposed based on the ring resonator filter in [22]. With this simple modification, the filter performance is improved, where four transmission poles (TPs) and six transmission zeros (TZs) can be realized. The analytical and accurate design equations for TPs and TZs are deduced using the transmission line theory. Then, based on the single-ended BPF structure, a balanced BPF circuit with high-selectivity DM filtering characteristic and high CM suppression is presented. The detailed analysis procedure, numerical calculation, and experimental measurements are demonstrated.

\section{The Proposed Single-Ended BPF}

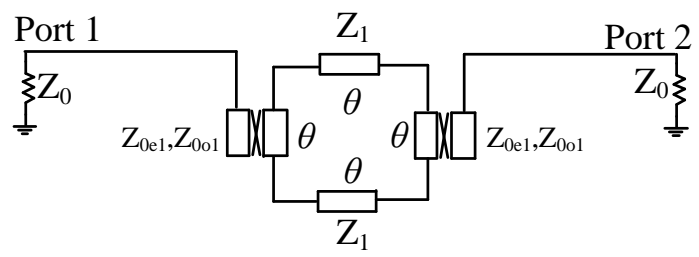

(a)

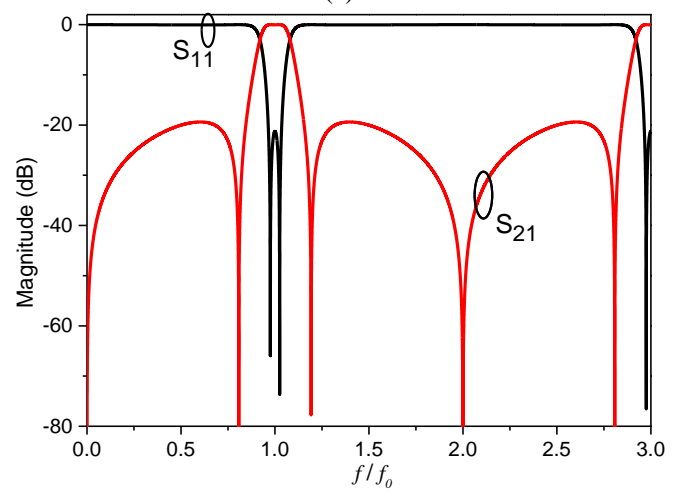

(b)

Fig. 1. (a) Ideal circuit of bandpass filter using a conventional dual-mode ring resonator, and (b) its S-parameter simulated results.

Fig. 1(a) shows a conventional bandpass filter using a dual-mode ring resonator fed by two quarter-wavelength 
side-coupled lines (characteristic impedances of $Z_{0 \mathrm{e} 1}$ and $Z_{0 \mathrm{ol}}$, electrical length of $\theta=90^{\circ}$ ), which has been analyzed in [22]. The simulated results of this filter using Ansoft Designer software are illustrated in Fig. 1(b), where two resonant modes within the passband and four TZs from 0 to $2 f_{0}$ ( $f_{0}$ denotes center frequency of the filter passband) can be obtained.

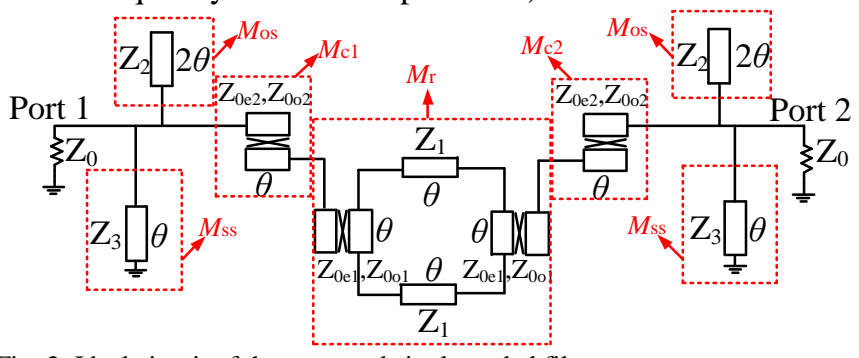

Fig. 2. Ideal circuit of the proposed single-ended filter.

Based on this conventional filter circuit diagram, two open coupled lines $\left(Z_{0 \mathrm{e} 2}, Z_{0 \mathrm{0o}}\right.$, electrical length $\left.\theta\right)$ with two open stubs $\left(Z_{2}\right.$, electrical length $\left.2 \theta\right)$ and two short loaded stubs $\left(Z_{3}\right.$, electrical length $\theta$ ) are inserted close to the input and output ports, as shown in Fig. 2, i.e., the ideal circuit of the proposed single-ended BPF. Accordingly, the simulated $S$-parameters of this ideal single-ended filter using Ansoft Designer software are illustrated in Fig. 3. When two open stubs (OSs) and two short stubs (SSs) are not loaded near the input/output ports, the filter structure is exactly the same as the filter in [22], where four TZs $\left(f_{\mathrm{tz} 1}, f_{\mathrm{tz} 3}, f_{\mathrm{tz} 4}\right.$, and $\left.f_{\mathrm{tz} 6}\right)$ from 0 to $2 f_{0}$ and four TPs within the passband can be achieved.

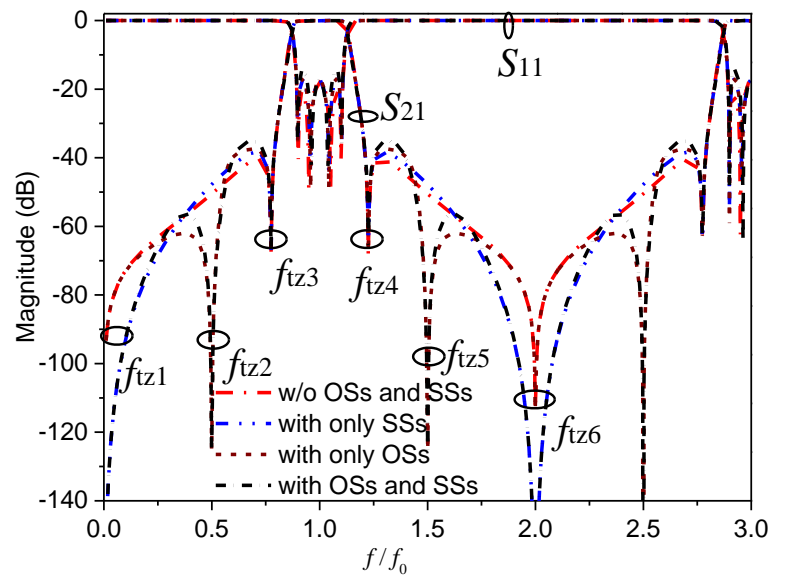

Fig. 3. Simulated results of Fig. 2 with/without open/short stubs $\left(Z_{1}=94 \Omega\right.$, $\mathrm{Z}_{2}=130 \Omega, \mathrm{Z}_{3}=27 \Omega, \mathrm{Z}_{0 \mathrm{el}}=143 \Omega, \mathrm{Z}_{0 \mathrm{01}}=78 \Omega, \mathrm{Z}_{0 \mathrm{e} 2}=130 \Omega, \mathrm{Z}_{002}=55 \Omega$, and $\left.\theta=90^{\circ}\right)$.

When two quarter-wavelength SSs are loaded between the input/output ports and coupled lines as shown in Fig. 2, the TZs at 0 and $2 f_{0}$ (i.e., $f_{\mathrm{tz} 1}$ and $f_{\mathrm{tz} 6}$ ) will be deeper, which can be seen in Fig. 3. Moreover, with these two SSs, it is easy to extend to construct a balanced filter, which will be seen in Section III. When two half-wavelength OSs are shunted to the input/output ports, two more TZs are introduced locating at $0.5 f_{0}$ and $1.5 f_{0}$ (i.e., $f_{\mathrm{tz} 2}$ and $f_{\mathrm{tz} 5}$ ), respectively, to improve the out-of-band suppression of the filter, while the positions of four TPs and the other four TZs $\left(f_{\mathrm{tz} 1}, f_{\mathrm{tz} 3}, f_{\mathrm{tz} 4}\right.$, and $\left.f_{\mathrm{tz} 6}\right)$ remain unchanged. Consequently, we can employ these two SSs and two OSs simultaneously to achieve four TPs and six TZs for selectivity improvement.

The two TZs $f_{\mathrm{tz} 2}$ and $f_{\mathrm{tz} 5}$ are not related to the characteristic impedances of OSs $Z_{2}$ and SSs $Z_{3}$, as illustrated in Fig. 4(a) and (b), respectively. However, once the OSs are not loaded, the two TZs $f_{\mathrm{tz} 2}$ and $f_{\mathrm{tz} 5}$ will not exist. If the characteristic impedance $Z_{2}$ is toward infinite, it can be represented as the unloaded OS status. Here, we set $Z_{2}$ as $20 \mathrm{M} \Omega$ to simulate the $S_{21}$ parameter, which is found that the $f_{\mathrm{tz} 2}$ and $f_{\mathrm{tz} 5}$ will disappear as shown in Fig. 4(a). For the variation of the characteristic impedance $Z_{3}$, as shown in Fig. 4(b), the TZs at 0 and $2 f_{0}$ will be deeper but other four $T Z s$ remain unchanged when $Z_{3}$ decreases from $67 \Omega$ to $27 \Omega$. If the characteristic impedance $Z_{3}$ increases to be infinite, it can be represented as the unloaded SS status, thus the curve will be the one with only OSs in Fig. 3.

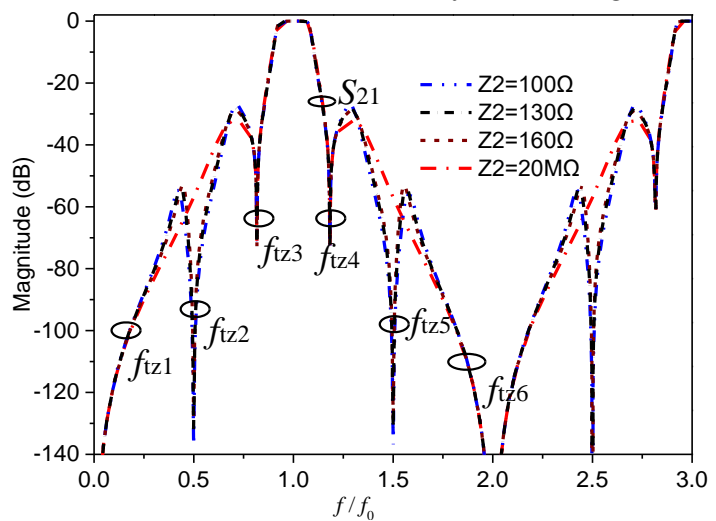

(a)

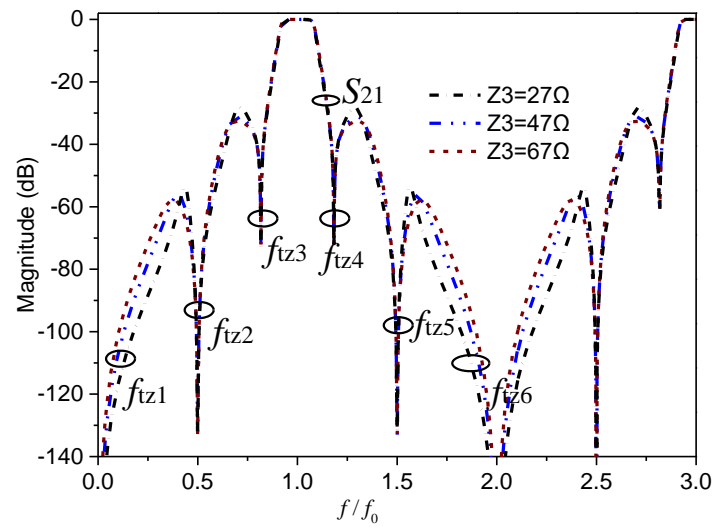

(b)

Fig. 4. Simulated $\left|S_{21}\right|$ results of Fig. 2 with (a) varied $Z_{2}$ and (b) varied $Z_{3}$.

The $A B C D$ matrix of the filter circuit in Fig. 2 can be defined as $M_{\text {ss }} \times M_{o s} \times M_{c 1} \times M_{r} \times M_{c 2} \times M_{o s} \times M_{\text {ss }}$, where $M_{\mathrm{ss}}$ and $M_{\text {os }}$ are the $A B C D$ matrices of the loaded quarter-wavelength short stub and half-wavelength open stub, respectively. Simply, the $A B C D$-matrices $M_{\mathrm{ss}}$ and $M_{o s}$ are as follows:

$$
\begin{gathered}
M_{\mathrm{ss}}=\left[\begin{array}{ll}
A_{\mathrm{ss}} & B_{\mathrm{ss}} \\
C_{\mathrm{ss}} & D_{\mathrm{ss}}
\end{array}\right]=\left[\begin{array}{cc}
1 & 0 \\
-j /\left(Z_{3} \tan \theta\right) & 1
\end{array}\right] \\
M_{\mathrm{os}}=\left[\begin{array}{ll}
A_{\mathrm{os}} & B_{\mathrm{os}} \\
C_{\mathrm{os}} & D_{\mathrm{os}}
\end{array}\right]=\left[\begin{array}{cc}
1 & 0 \\
j \tan 2 \theta / Z_{2} & 1
\end{array}\right] .
\end{gathered}
$$

The $A B C D$ matrices of the open coupled lines $M_{c 1}$ and $M_{c 2}$ can be expressed as [23] 


$$
M_{\mathrm{c} 1}=M_{\mathrm{c} 2}=\frac{1}{n}\left[\begin{array}{cc}
m & (m+n)(m-n) \\
1 & m
\end{array}\right]
$$

where $m=-\frac{j\left(Z_{0 e 2}+Z_{002}\right) \cot \theta}{2}, n=-\frac{j\left(Z_{0 e 2}-Z_{002}\right) \csc \theta}{2}$. On the other hand, the $A B C D$ matrix of the conventional bandpass filter using dual-mode ring resonator $M_{r}$ can be converted from its admittance $(Y)$-matrix, while the $Y$-matrix $Y r$ can be directly obtained as [22]

$$
Y_{r}=\left[\begin{array}{ll}
Y_{r 11} & Y_{r 12} \\
Y_{r 12} & Y_{r 11}
\end{array}\right]
$$

where

$$
\begin{aligned}
& Y_{r 11}=\frac{\left(B-Y_{u e}{ }^{2} Z_{1}^{2}\right)\left(1+Y_{u e} Z_{1}\right) \cos \theta}{j B Z_{1} \sin \theta} \\
& Y_{r 12}=\frac{B+Y_{u e} Z_{1}^{2}}{-j B Z_{1} \sin \theta} \\
& B=\left(2 Y_{u e} Z_{1}+Y_{u e}{ }^{2} Z_{1}^{2}\right) \cos ^{2} \theta-\sin ^{2} \theta \\
& Y_{u e}=Y_{11}-\frac{Y_{12}}{Y_{11}} \\
& Y_{11}=\frac{Z_{0 o 1}+Z_{0 e 1}}{2 Z_{0 o 1} Z_{0 e 1}} \\
& Y_{12}=\frac{Z_{0 e 1}-Z_{0 o 1}}{2 Z_{0 o 1} Z_{0 e 1}} .
\end{aligned}
$$

Finally, after the conversions between $A B C D$-, admittance $Y$ and scattering $S$-matrices, we can obtain the $S$-matrix of the whole filter circuit in Fig. 2. The locations of TZs can be determined when $S_{21}=0$. Six TZs can be solved and expressed as:

$$
\begin{aligned}
& \theta_{t z 1}=0, \quad \theta_{t z 6}=\pi \\
& \theta_{t z 2}=\pi / 4, \quad \theta_{t z 5}=3 \pi / 4 \\
& \theta_{t z 3}=\arccos \sqrt{\frac{Z_{0 e 1}+Z_{0 o 1}-2 Z_{1}}{Z_{0 e 1}+Z_{0 o 1}+2 Z_{1}}}, \quad \theta_{t z 4}=\pi-\theta_{t z 3}
\end{aligned}
$$

where $f_{t z \mathrm{i}}=2 \theta_{t \mathrm{zi}} f_{0} / \pi, \mathrm{i}=1,2, \ldots, 6$.

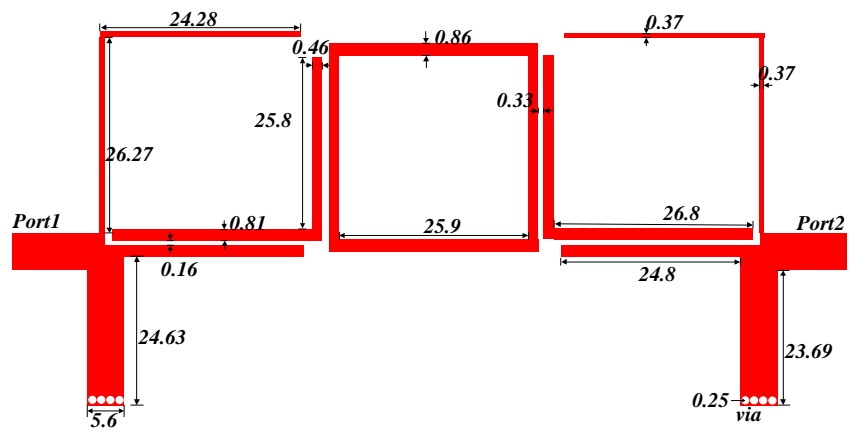

(a)

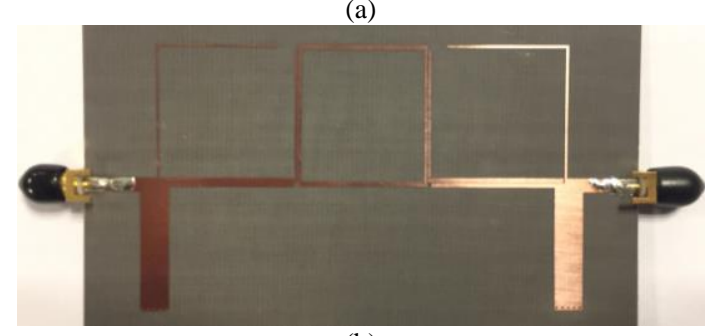

(b)

Fig. 5. (a) Layout with physical dimensions (unit: $\mathrm{mm}$ ) and (b) fabricated photograph of the proposed single-ended BPF.

In order to verify the proposed design theory, a single-ended BPF with the center frequency at $2.1 \mathrm{GHz}$ is fabricated on a dielectric substrate with the relative permittivity of 2.65 and thickness of $1 \mathrm{~mm}$. Fig. 5 illustrates a verified physical layout of the proposed single-ended filter and its fabricated photograph. As illustrated in Fig. 6, the measured 3-dB fractional bandwidth is $23.7 \%$ (from 1.82 to $2.31 \mathrm{GHz}$ ). The measured insertion losses are less than $1.6 \mathrm{~dB}$ and return losses are better than $15 \mathrm{~dB}$ within the passband. There are four TPs in the measured results located at 1.87, 2.0, 2.23, and $2.25 \mathrm{GHz}$. Besides, six measured TZs are located at 0, 1.14, 1.55, 2.43, 3.1, and $4.16 \mathrm{GHz}$, respectively. The simulated frequency responses using HFSS software and measured counterparts are basically in good agreement. Fig. 7 illustrates the current distributions at four resonant frequencies of 1.87, 2.0, 2.23, and $2.25 \mathrm{GHz}$. In Fig. 7(a) and (d), the first and fourth resonances created by coupled lines with multiple stubs generate the RF signal path from source to load ports. Similarly, as shown in Fig. 7(b) and (c), the second and third resonances are generated by the dual-mode ring resonator associate with the first and fourth resonances to form the RF signal path from source to load ports. These four resonances are excited simultaneously to constitute a passband for the wideband filter implement. With the help of TZs, good frequency selectivity and high stopband rejection of the filter can be well achieved.

Table 1 tabulates the performance comparisons of the proposed BPF with some previous reported works, and it can be seen that the presented study has a wide passband response and multiple TZs to realize good out-of-band rejection.

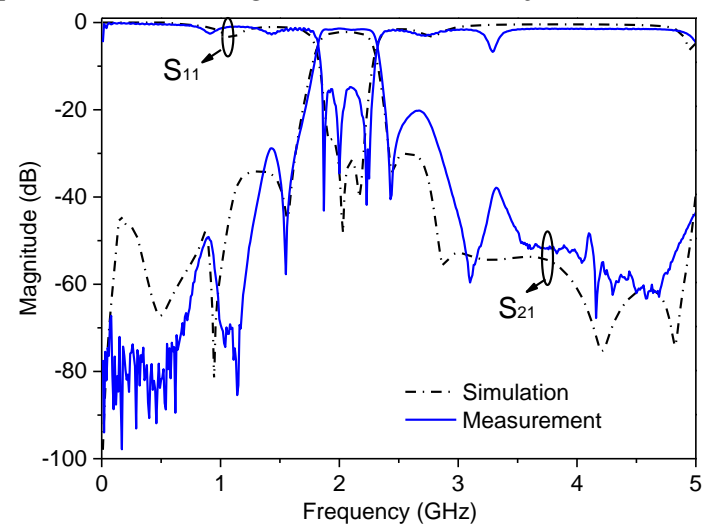

Fig. 6. Simulated and measured S-parameters of the proposed single-ended BPF.

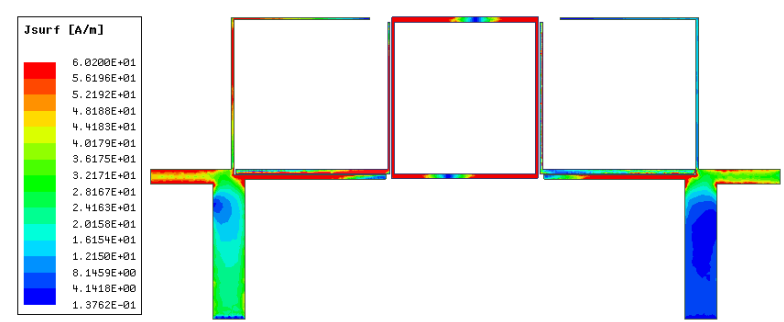

(a)

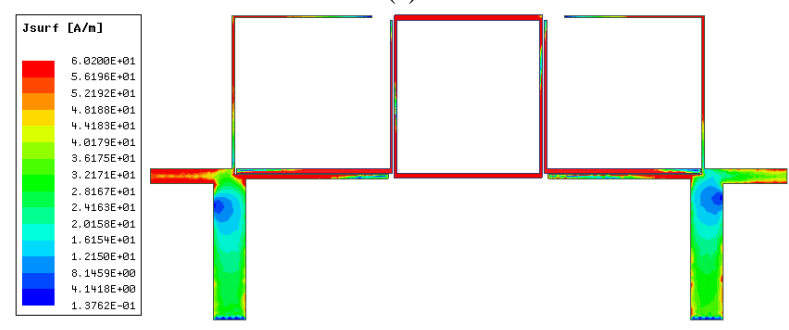


(b)

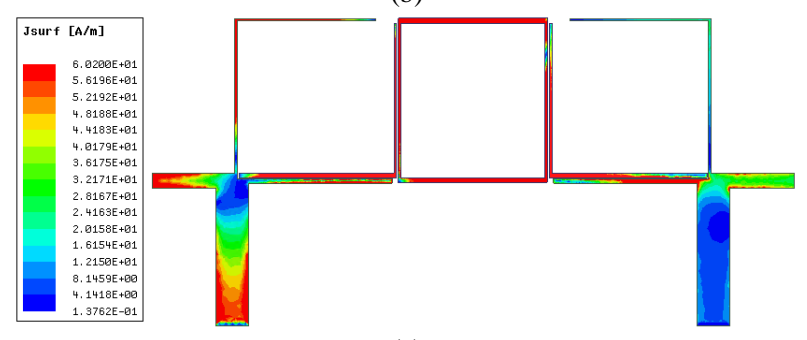

(c)

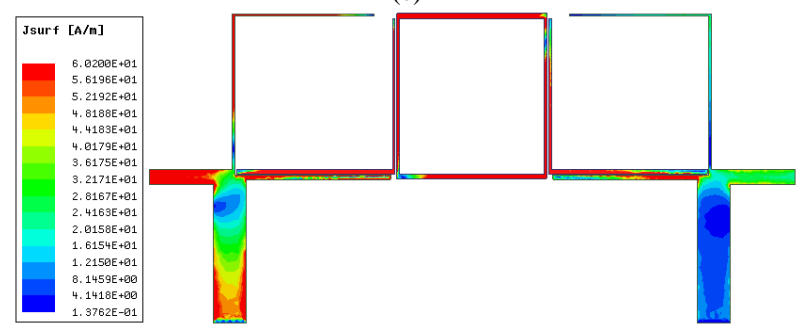

(d)

Fig. 7. Simulated current distributions at (a) 1.87, (b) 2.0, (c) 2.23, and (d) 2.25 GHz.

Table 1. Comparisons with some previous single-ended filters

\begin{tabular}{|c|c|c|c|c|}
\hline Ref. & $\begin{array}{c}\text { Center } \\
\text { frequency }\left(f_{0}\right)\end{array}$ & $\begin{array}{c}\text { 3-dB fractional } \\
\text { bandwidth }\end{array}$ & $\begin{array}{c}\text { Passband } \\
\text { orders }\end{array}$ & TZs \\
\hline$[5]$ & $1.98 \mathrm{GHz}$ & $15.2 \%$ & 3 & 4 \\
\hline$[6]$ & $1.39 \mathrm{GHz}$ & $10.0 \%$ & 4 & 3 \\
\hline$[9]$ & $1.93 \mathrm{GHz}$ & $78 \%$ & 4 & 4 \\
\hline$[10]$ & $9 \mathrm{GHz}$ & $5.5 \%$ & 3 & 4 \\
\hline $\begin{array}{c}\text { This } \\
\text { work }\end{array}$ & $2.1 \mathrm{GHz}$ & $23.7 \%$ & 4 & 6 \\
\hline
\end{tabular}

\section{The Proposed BALANCED BPF}

By doubling the above mentioned single-ended BPF and removing the grounding vias, a balanced $\mathrm{BPF}$ can be proposed. Fig. 8 shows the ideal circuit of the proposed balanced BPF as well as its DM and CM equivalent circuits. The whole circuit in Fig. 8(a) is ideally symmetrical with respect to the center red line. Under the DM or CM excitation, a virtual shorted circuit (electric wall) or open circuit (magnetic wall) appears along the symmetric line, respectively. The DM equivalent circuit in Fig. 8 (b) is actually the same as the above single-ended BPF. Considering the suppression characteristic of the $\mathrm{CM}$ equivalent circuit in Fig. 8(c), the values of characteristic impedances are slightly adjusted based on the parameters of the single-ended filter.

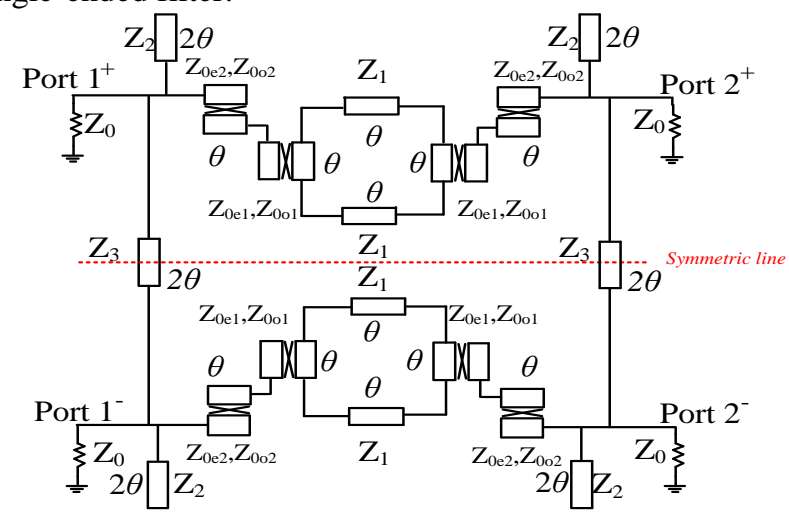

(a)

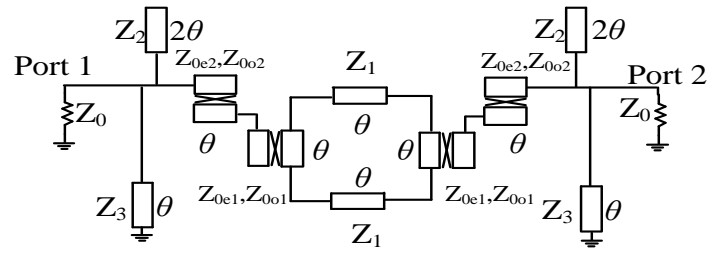

(b)

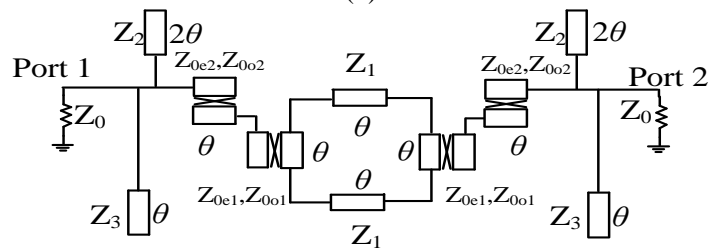

(c)

Fig. 8. (a) The ideal circuit of the proposed balanced filter, (b) DM equivalent circuit, and (c) $\mathrm{CM}$ equivalent circuit.

For demonstration, the balanced BPF example designed on the same substrate as above is fabricated, whose center frequency is at $2.1 \mathrm{GHz}$. The layout with physical dimensions and fabricated photograph are shown in Fig. 9. Fig. 10 illustrates the measured DM and CM $S$-parameters along with the simulations for comparisons. In Fig. 10(a), the measured 3-dB DM fractional bandwidth is $24.7 \%$ (from 1.81 to 2.32 $\mathrm{GHz}$ ). Within the DM passband, the measured insertion losses are less than $1.0 \mathrm{~dB}$ and return losses are better than $12 \mathrm{~dB}$. There are six DM TZs in the measured results located at $0,1.14$, $1.56,2.43,3.19$, and $4.18 \mathrm{GHz}$. Besides, three measured TPs are located at 1.88, 1.98, and $2.23 \mathrm{GHz}$, respectively. As shown in Fig. 10(b), the measured CM suppression of the balanced $\mathrm{BPF}$ is greater than $20 \mathrm{~dB}$ from 0.5 to $5 \mathrm{GHz}$. Note that the measured CM suppression at the center frequency of $2.1 \mathrm{GHz}$ is over $44.5 \mathrm{~dB}$. Observed from Fig. 10, the full-wave electromagnetic simulations and measurements are almost in good agreement. The slight discrepancy between the simulated and measured frequency responses may be attributed to the fabrication and measurement errors.

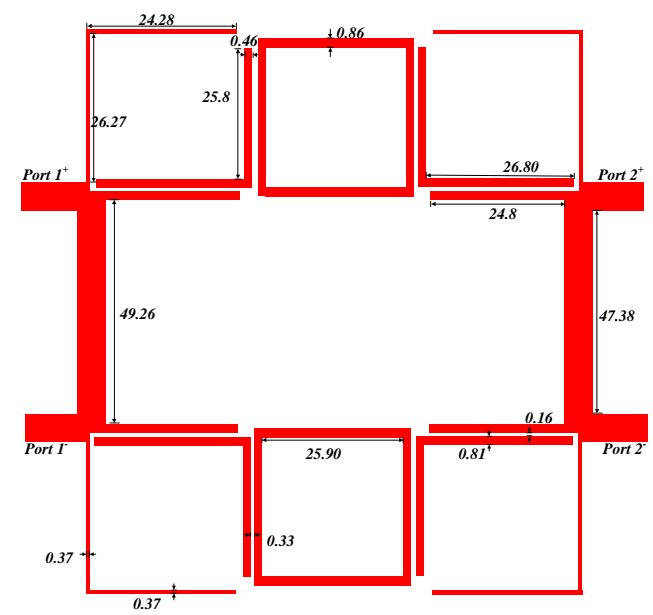

(a) 


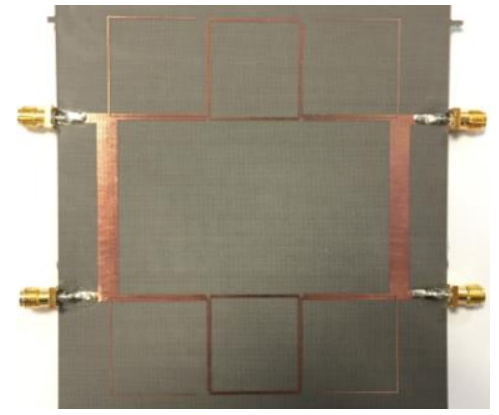

(b)

Fig. 9. (a) Layout with physical dimensions (unit: $\mathrm{mm}$ ) and (b) fabricated photograph of the proposed balanced BPF.

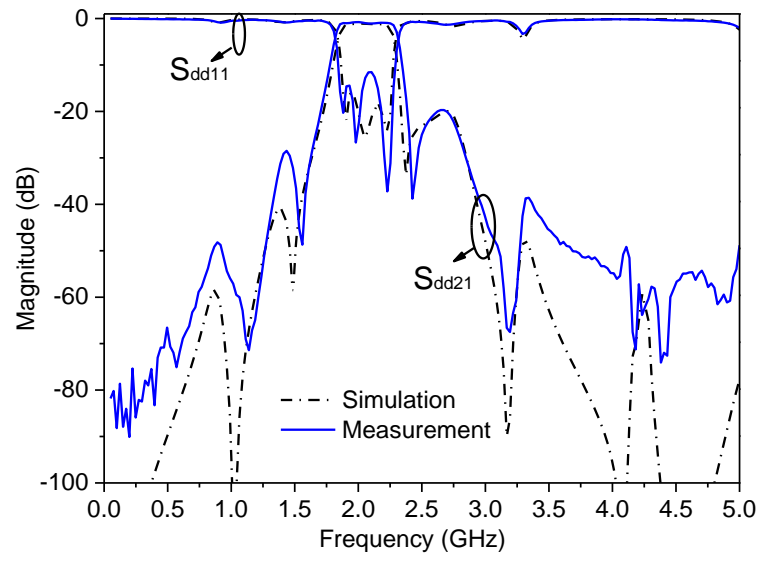

(a)

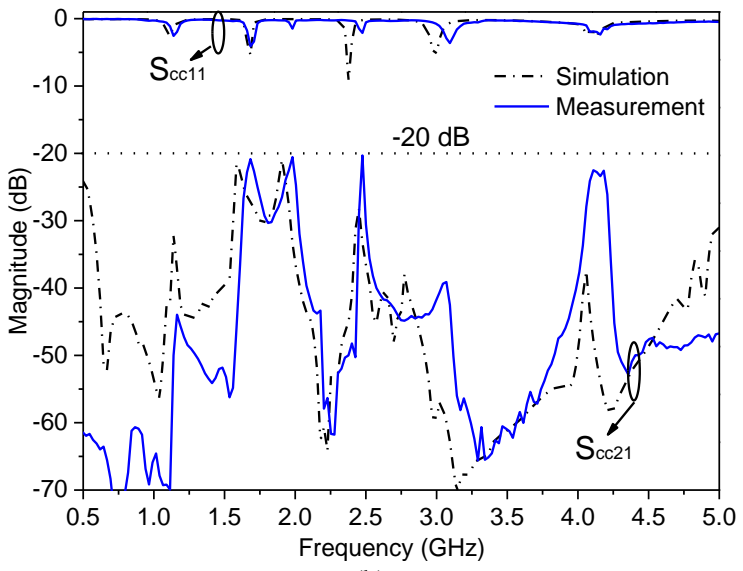

(b)

Fig. 10. Simulated and measured S-parameters of the balanced BPF.

\section{CONCLUSION}

Two high-selectivity bandpass filters using dual-mode ring resonators loaded with multiple stubs have been presented in this paper. The analytical procedure for calculating the TZs' positions of the single-ended BPF is provided. Then, the balanced BPF is proposed by doubling the single-ended BPF without any grounding vias. The validity of these two filters is verified by the fabrications and experiments. The proposed single-ended BPF with simple structure features high selectivity and deep stop-band suppression with the help of six TZs. The advantages of the proposed balanced BPF include simple configuration, good insertion losses and high selectivity within DM passband, high DM suppression at the out-of-band, and all-frequency high CM suppression. It can be expected that these two BPFs are attractive for applications in the modern wireless communication systems.

\section{REFERENCES}

[1] M. Forouzanfar and M. Joodaki, "Systematic design of hybrid high power microwave amplifiers using large gate periphery GaN HEMTs," AE $\ddot{U}$ - Int. J. Electron. Commun., vol. 84, pp. 225-233, 2018.

[2] Z. Zhuang, Y. Wu, and Y. Liu, "Dual-band filtering out-of-phase balanced-to-single-ended power divider with enhanced bandwidth," $A E \ddot{U}$ Int. J. Electron. Commun., vol. 82, pp. 341-345, 2017.

[3] K. D. Xu, F. Zhang, Y. Liu, and Q. H. Liu, "Bandpass filter using three pairs of coupled lines with multiple transmission zeros," IEEE Microw. Wireless Compon. Lett., vol. 28, no. 7, pp. 576-578, 2018.

[4] J. Ai, Y. Zhang, K. D. Xu, D. Li, and Q. H. Liu, "Design of a high-selectivity quad-band bandpass filter based on $\lambda / 4$ resonators with alternative J/K inverters," AE ̈̈ - Int. J. Electron. Commun., vol. 70, pp. 1028-1033, 2016.

[5] J. Li, Y. Huang, G. Wen, X. Xue, and J. Song, "Compact and high-selectivity microstrip bandpass filter using two-stage twist-modified asymmetric split-ring resonators," Electron. Lett., vol. 51, no. 8, pp. 635-637, 2015.

[6] R. Gómez-García and J. I. Alonso, "Design of sharp-rejection and low-loss wide-band planar filters using signal-interference techniques," IEEE Microw. Wireless Compon. Lett., vol. 15, no. 8, pp. 530-532, Aug. 2005.

[7] M. Sánchez-Renedo, and R. Gómez-García, "A class of highselectivity microstrip transversal bandpass filter using non-resonating nodes," 40th European Microwave Conf., Paris, pp. 292-295, 2010.

[8] W. J. Feng, X. Gao, W. Q. Che, and Q. Xue, "Bandpass filter loaded with open stubs using dual-mode ring resonator," IEEE Microw. Wireless Compon. Lett., vol. 25, no. 5, pp. 295-297, May 2015.

[9] K. D. Xu, F. Y. Zhang, Y. H. Liu, and W. Nie, "High selectivity seventh-order wideband bandpass filter using coupled lines and open/shorted stubs," Electron. Lett., vol. 54, no. 4, pp. 223-225, 2018.

[10] P.-J. Zhang, and M.-Q. Li, "Cascaded trisection substrate-integrated waveguide filter with high selectivity," Electron. Lett., vol. 50, no. 23, pp. 1717-1719, 2014.

[11] W. Feng, W. Che, and Q. Xue, "The proper balance: overview of microstrip wideband balance circuits with wideband common mode suppression," IEEE Microw. Mag., vol. 16, no. 5, pp. 55-68, Jun. 2015.

[12] C. H. Wu, C. H. Wang, and C. H. Chen, "Stopband-extended balanced bandpass filter using coupled stepped-impedance resonators," IEEE Microw. Wireless Compon. Lett., vol. 17, no. 7, pp. 507-509, Jul. 2007.

[13] J. Shi, C. Shao, J. X. Chen, Q. Y. Lu, Y. Peng, and Z. H. Bao, "Compact low-loss wideband differential bandpass filter with high common-mode suppression," IEEE Microw. Wirel. Compon. Lett., vol. 23, no. 9, pp. 480-482, 2013.

[14] W. Feng, W. Che, and Q. Xue, "Balanced filters with wideband common mode suppression using dual-mode ring resonators," IEEE Trans. Circuits Syst. I, Regular Papers, vol. 62, no. 6, pp. 1499-1507, Jun. 2015.

[15] X. H. Wu, and Q. X. Chu, "Compact differential ultra-wideband bandpass filter with common-mode suppression," IEEE Microw. Wirel. Compon. Lett., vol. 22, no. 9, pp. 456-458, 2012.

[16] W. Feng, X. Gao, W. Che, W. Yang, and Q. Xue, "High selectivity wideband balanced filters with multiple transmission zeros," IEEE Trans. Circuits Syst. II, Exp. Briefs, vol. 64, no. 10, pp. 1182-1186, Oct. 2017.

[17] R. Gomez-Garcia, R. Loeches-Sanchez, D. Psychogiou, D. Peroulis, "Multi-stub-loaded differential-mode planar multi-band bandpass filters," IEEE Trans. Circuits Syst. II, Exp. Briefs, vol. 65, no. 3, Mar. 2018.

[18] B. Zhang, Y. Wu, and Y. Liu, "Wideband single-ended and differential bandpass filters based on terminated coupled line structures," IEEE Trans. Microw. Theory Techn., vol. 65, no. 3, pp.761-774, Mar. 2017.

[19] J. Shi, Z. Shen, J. Qiang and Q. Cao, "Compact wideband-balanced bandpass filter with high-selectivity and wideband CM suppression," Electron. Lett., vol. 54, no. 1, pp. 32-33, 2018.

[20] Q.-X. Chu and L.-L. Qiu, "Wideband balanced filters with high selectivity and common-mode suppression," IEEE Trans. Microw. Theory Techn., vol. 63, no. 10, pp. 3462-3468, Oct. 2015.

[21] W.-J. Zhou and J.-X. Chen, "High-selectivity tunable balanced bandpass filter with constant absolute bandwidth," IEEE Trans. Circuits Syst. II, Exp. Briefs, vol. 64, no. 8, pp. 917-921, Aug. 2017.

[22] M. K. M. Salleh, G. Prigent, O. Pigaglio, and R. Crampagne, "Quarter-wavelength side-coupled ring resonator for bandpass filters," 
IEEE Trans. Microw. Theory Techn., vol. 56, no. 1, pp. 156-162, Jan. 2008.

[23] G. Matthaei, L. Young, and E. M. T. Jones, Microwave Filters, Impedance Matching Networks and Coupling Structures. Norwood, MA, USA: Artech House, 1985, chapter 5, sec. 5, pp. 221. 